\title{
Modeling of radiative energy transfer dynamics
}

\author{
T.L. LAZARUS, G. ARMAGAN* and N.P. BARNES
}

NASA Langley Research Center, MS 474, Hampton, VA 23681, U.S.A.

* The College of William and Mary, Department of Physics, Williamsburg, VA 23187, U.S.A.

\begin{abstract}
A comprehensive model which can predict the $\mathrm{Ho}^{3+5} \mathrm{5}_{7} \mathrm{YAG}\left(\mathrm{Y}_{3} \mathrm{Al}_{5} \mathrm{O}_{12}\right)$ manifold luminescence and radiative energy transfer rate among $\mathrm{Ho}$ ions for a given concentration and emission path length within the crystal has been developed. Independently, a model was devised to calculate these rates using measured lifetimes as well as calculated radiative and non-radiative lifetimes. The rates of radiative energy transfer were determined from both of these models and found to be in close agreement.
\end{abstract}

\section{Introduction.}

An extensive characterization of this YAG manifold has been conducted. This study includes absorption, emission, and lifetime measurements using a wide range of Ho concentrations (from 0.32 to $8.5 \%$ ), temperatures (from 30 to $500 \mathrm{~K}$ ), and emission path lengths of the $2 \mu \mathrm{m}$ Ho emission. The lifetime of this manifold has been observed to increase with concentration and decrease with temperatures below $295 \mathrm{~K}$ in contrast to most other Lanthanide series elements. Rates of radiative energy transfer were calculated independently from luminescence and lifetime models derived from these measurements. The close agreement in these rates suggests that strong radiative energy transfer among excited Ho ions in the ${ }^{5} I_{7}$ manifold is responsible for the observed behavior of Ho lifetimes.

\section{Experimentation.}

Ho:YAG crystals in concentrations of $0.32,1,3$, and $8.5 \%$ were obtained from A.A. Kaminskii from the Institute of Crystallography at the Russian Academy of Science. These four samples were then cut and polished into rectangular cross section slabs, each with length $8.73 \mathrm{~mm}$, width $2.10 \mathrm{~mm}$, and height $2.62 \mathrm{~mm}$.

Absorption measurements of the $2 \mu \mathrm{m}$ Ho manifold were made as a function of concentration and temperature.

Luminescence and lifetime

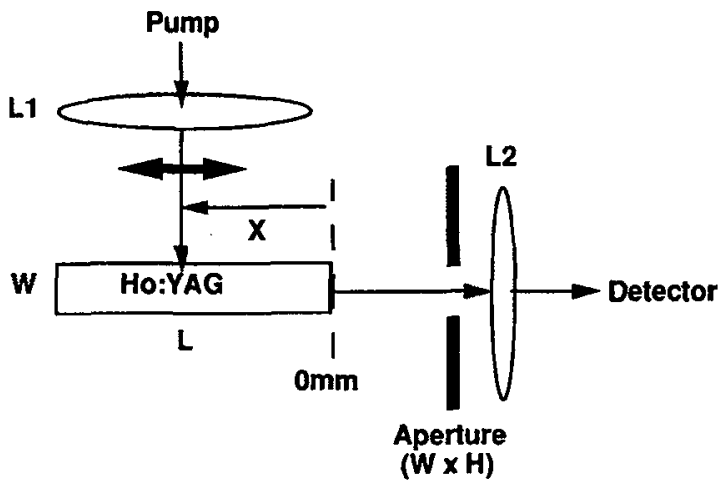

Fig 1. Definition of "emission path length," $x$ measurements of the $2 \mu \mathrm{m}$ Ho manifold were oblained as functions of concentration, emission path length (see Fig 1), and temperature. Luminescence was studied under the excitation of the ${ }^{5} \mathrm{~F}_{3}$ manifold using a $\mathrm{cw}$ argon ion laser emitting at $460 \mathrm{~nm}$. Lifetimes were acquired under the excitation of the ${ }^{5} S_{2}$ manifold using a pulsed dye laser emitting at $542 \mathrm{~nm}$. 


\section{Luminescence Model.}

The following model calculates the $\mathrm{Ho}^{5}{ }_{7}$ luminescence and radiative energy transfer rate among Ho ions for a given concentration, emission path length, and temperature. Table 1 below lists the significant parameters and equations in the order of their derivation in the model.

$$
\begin{aligned}
& \mathrm{I}_{\mathrm{O}}(\lambda) \quad=\text { luminescence spectrum at the pump position nearest the detector } \\
& \mathrm{I}_{0}^{+}(\lambda), \mathrm{I}_{0}^{-}(\lambda)=\mathrm{I}_{0}(\lambda) \\
& =\text { initial hemispherical emission within the slab at } X \text { after absorption of } \\
& \kappa=\text { fraction of } \mathrm{I}_{0}^{+}(\lambda) \text { or } \mathrm{I}_{0}^{-}(\lambda) \text { totally internally reflected within the } \\
& \text { slab } \\
& =0.4499 \text { for YAG at } 2.1 \mu \mathrm{m}
\end{aligned}
$$

$R=$ reflection coefficient for the end of the sample nearest the detector$$
=0.0821 \text { for } Y A G \text { at } 2.1 \mu \mathrm{m}
$$

$\alpha(\lambda)=$ absorption coefficient of Ho:YAG

$d=$ variably small average distance over which emission can be transmitted or reabsorbed

$=0.1 \mathrm{~mm}$

$\Gamma^{+}(\lambda), \mathrm{I}^{-}(\lambda)=$ diminished initial emission toward, away from the detector

$$
\begin{aligned}
\mathrm{X}^{+}, \mathrm{X}^{-} & =\text {total path length } \mathrm{I}^{+}(\lambda), \mathrm{I}^{-}(\lambda) \text { travels before it is detected } \\
\mathrm{I}(\lambda) & =\mathrm{I}^{+}(\lambda)+\mathrm{I}^{-}(\lambda) \\
& =\text { total detected luminescence due to absorption of pump radiation } \\
& \text { at } X \\
& =K I_{0}(\lambda)(1-R) B(\lambda) C(\lambda) \\
B(\lambda) & =1+R A^{n}+R^{2} A^{2 n}+R^{3} A^{3 n}+R^{4} A^{4 n} \\
C(\lambda) & =A^{n+}+A^{n-} \\
A(\lambda) & =e^{-\alpha(\lambda) d}+K\left(1-e^{-\alpha(\lambda) d}\right. \\
n, n^{+}, n^{-} & =2 L / d, X / d,(2 L-X) / d \quad(L=\text { slab length) }
\end{aligned}
$$

\section{Table 1}

An example of the calculated $\mathrm{Ho}^{5} \mathrm{I}_{7}$ luminescence as compared to the experimental is shown in Fig 2 for a moderate concentration of $3 \%$, temperature of $295 \mathrm{~K}$, and emission path length of $4.3 \mathrm{~mm}$. 
The $\mathrm{H}_{0}{ }^{5} \mathrm{I}_{7}$ radiative energy transfer rate as a function of temperature can be derived from the parameters and equations listed in Table 1. The portion of the initial luminescence emission toward the detector remaining inside the crystal after a distance $d$ is given by

$$
+(\lambda, d)=\kappa I_{0}^{+}(\lambda)\left[e^{-\alpha(\lambda) d}+\kappa\left(1-e^{-\alpha(\lambda) d}\right)\right] .
$$

The first term represents the fraction of the initial emission toward the detector that is totally internally reflected (TIR) and remaining after the distance $d$. The second term represents the fraction of this initial TIR emission that is reabsorbed over $\mathrm{d}$, reemitted, and totally internally reflected toward the detector. Therefore $d$ is the path length inside the crystal length over which one TIR transmission, reabsorption, and reemission takes place. The fraction of the initial TIR emission participating in radiative energy transfer (reabsorption - reemission of $2 \mu \mathrm{m}$ photons) over $d$ is therefore given by

$$
i_{\operatorname{rad}}(\lambda, d)=\kappa b_{0}^{+}(\lambda)\left[\kappa\left(1-e^{-\alpha(\lambda) d}\right)\right] .
$$

This quantity can then be transiormed such that it is proportional to and has the same units as the radiative energy transfer rate:

$$
\begin{aligned}
\Gamma_{\operatorname{rad}}(\lambda, d) & =\text { energy / [ (area) (time) }] \\
& =\text { (energy / \#photon) (\#photon / time) }(1 / \text { area) } \\
& =\text { (energy / \#photon) }\left(1 / \text { area) } W_{\text {rad }}\right. \\
& =\left(E^{\prime} / \text { A) } W_{\text {rad }}\right.
\end{aligned}
$$

where $E^{\prime}=C(0.59 \mathrm{eV} / 2.1 \mu \mathrm{m}$ photon $)$, and $C$ is a normalization constant. With reference to $F i g 3, A$ is the area of the portion of the sphere centered at the point of initial emission $(X, H / 2, W / 2)$ that is subtended by $\mu \mathrm{TIR}$, the solid angle of TIR, and the sides of the slab. After the appropriate geometrical analysis and given that the pump spot radius $\ll L, W, H$ (slab dimensions), it was found that $A \sim 0.08 \mathrm{~cm}^{2}$. Thus,

$$
W_{\text {rad }}{ }^{\prime}=C^{-1}(0.59)^{-1}(0.08) \kappa^{2} I_{0}^{+}(\lambda)\left(1-e^{-\alpha(\lambda) d}\right),
$$

and the actual radiative energy transfer rate is,

$$
W_{\mathrm{rad}}=\left(\lambda_{2}-\lambda_{1}\right)^{-1} \int_{\lambda 1} \lambda 2 \mathrm{w}_{\mathrm{rad}} \mathrm{d} \lambda \text {, }
$$

where $\lambda_{1}=1.82 \mu \mathrm{m}, \lambda_{2}=2.15 \mu \mathrm{m}$.

\section{Lifetime Model.}

The $\mathrm{Ho}^{5}{ }^{5}$ radiative energy transfer rate may be calculated from the following equation depicting the observed behavior of the corresponding lifetimes. For a given temperature,

$$
1 / \tau_{\text {meas }}=1 / \tau_{\text {rad }}+1 / \tau_{\text {non-rad }}-W_{\text {rad }}
$$

where $\tau_{\text {meas }}$ is the measured lifetime, $\tau_{\text {rad }}$ is the radiative transition lifetime (given as $6.3 \mathrm{~ms}^{1}$ ), $w_{\text {rad }}$ is the radiative energy transfer rate, and $1 / \tau_{\text {non-rad }}$ is the non-radiative transition rate ${ }^{2}$ given by 


$$
1 / \tau_{\text {non-rad }}=10^{7}(1+m)^{p} e^{-\beta(p-2.4)}
$$

where $\beta=2, p$ is the smallest number of the highest frequency lattice phonons of energy hv (the release of which causes the non-radiative transition across the energy gap phv), and $m$ is the thermal occupancy of phonons of frequency $v$.

Examples of the $\mathrm{Ho}^{5}{ }_{7}$ radiative energy transfer rates calculated from both the luminescence and lifetime models as functions of temperature are shown in comparison in Fig 4. The constant $\mathrm{C}$ in the rate determined from the luminescence model was selected such that the rate from the luminescence model was normalized with respect to that from the lifetime model.

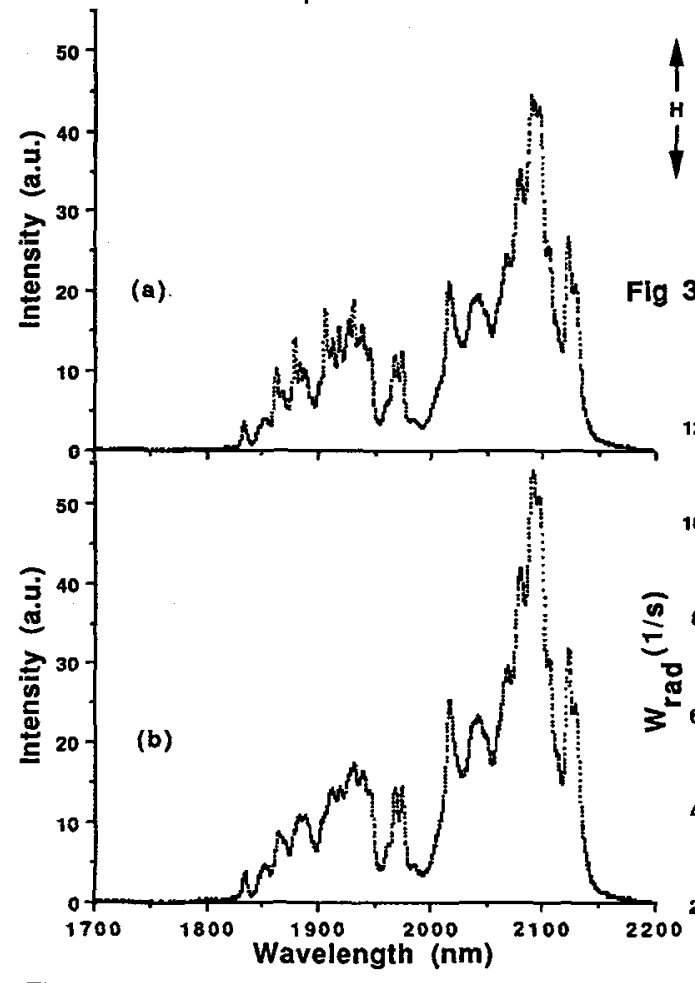

Fig 2. Theoretical (a) and measured (b) luminescence spectra for YAG: Ho(3 \%),295 K, and $X=4.3 \mathrm{~mm}$.
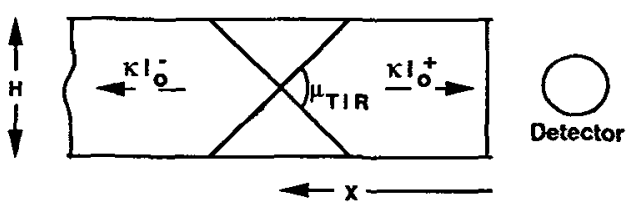

Fig 3. Total Internal reflection with respect to slab geometry.

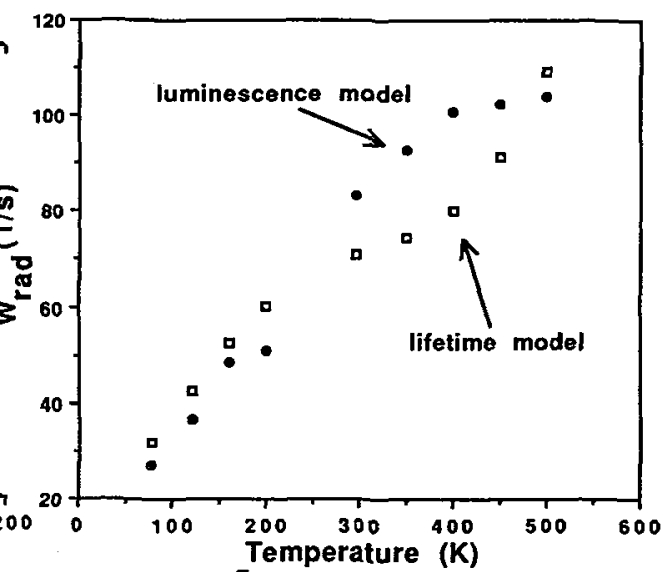

Fig 4. The $\mathrm{Ho}^{5} \mathrm{I}_{\mathbf{7}}$ radiative energy transfer rate temperature dependence for YAG:Ho(3 \%), $X=4.3 \mathrm{~mm}$.

\section{Conclusions.}

Two models were developed for the study of radiative energy transfer dynamics through both spectral (luminescence emission and absorption) and kinetic (lifetime) measurements. The spectral and kinetic measurements were correlated through close agreement in the temperature dependence of radiative energy transfer rates determined independently from the respective models.

\section{References.}

1. G. Armagan, to be published.

2. C.J. Donnelly and G.F. Imbusch in: Advances in Nonradiative Processes in Solids (Ed. B. Di Bartolo) Plenum Press, New York (1991). 